\title{
Numerical and experimental testing of normality in the functioning of the bearings system
}

\author{
A. Leopa*, S. Nastac and C. Debeleac \\ Research Center for Mechanics of the Machines, and Technological Equipments, Faculty of Engineering, \\ "Dunarea de Jos" University of Galati, Braila, Romania
}

\begin{abstract}
A highly efficient seismic protection of bridges have been demonstrated by the passive control systems with mechanical links with selective viscoelastic energy dissipation through viscous friction and dry friction. These systems are able to take over the deformations and movements caused by temperature variations, those due to seismic activities and from road traffic. A number of natural factors such as atmospheric and anthropogenic parameters, such as shock and vibration from road traffic, lead to the degradation of the viscoelastic bonds of passive control systems based on rubber molding. The presence of the elastic and dissipative nonlinear forces in the functioning of antiseismic systems inevitably leads to quantitative and qualitative changes of the structural responses of bridges and viaducts both in time and spectral domains. The implications of these changes on the integrity and stability of the bridge or viaduct sections may be from the simplest to the most severe depending on the type of seismic excitation to which they are subjected. For these reasons, it is imperative that we should identify and quantify the degree of nonlinearity of antiseismic systems embedded in the structure of bridges and viaducts in order to prevent accidents with severe socioeconomic implications. In this way can determine the dependence of the nonlinear behavior of the bearing system and the degree of degradation of their viscoelastic links. From a theoretical point of view the following parameters of the vibration of the system will be comparatively analyzed: the displacement and acceleration of the bridge deck in time and frequency domains, the hysteretic loop, the representation in the plane of phases, the power spectral density and the spectrograms of the acceleration signals. From an experimental point of view there will be identified and plotted the parameters able to provide information regarding the abnormal operation of the bearings from the elastomer: system acceleration, representations in time and frequency and its spectrogram.
\end{abstract}

Keywords: Dynamic, viscoelastic, vibration, nonlinear, rubber

\section{Introduction}

Bridges are vital structures designed to provide road and rail links over natural or artificial obstacles. For this reason, these structures must be protected both against actions coming from seismic activity, as well as against the loadings arising from road and rail traffic. In order to prevent partial or total destruction of bridges and viaducts under the above mentioned loadings, it is necessary to embed certain systems in their structure, designed to reduce or eliminate all undesirable effects induced by perturbations. An example of such systems, which are used in bridges and viaducts in the isolation and energy dissipation introduced in the structure, may be the elements of molding rubber. Such dynamic isolation systems have been used as the pillars of the bridge deck on the motorway A3 cells from Transylvania in Romania, the viaduct located at km $29+602,75 \leftrightarrow 29+801,25$ (at Săvădisla between Târgu Mureş and Cluj) [1]. Due to seismic activities, road traffic and the action of atmospheric factors, the rubber changes

*Corresponding author. E-mail: leopa.adrian@ugal.ro. 
its properties which leads to the modification of the dynamic response of the bridge to impulsive actions. Please replace it as: It was necessary to replace the systems in order to avoid partial or total degradation of the bridge.

This study proposes a methodology for assessing the condition of normal operation of a viaduct system bearing for the following reasons:

- developing a predictive maintenance of the anti-seismic systems, allowing them to change the optimum time;

- post-seismic diagnosis of bearing systems based on their dynamic response to impulsive loading.

Resistance structure supports, on each direction of travel, a roadway width of $12.00 \mathrm{~m}$ which includes two lanes each being $3.75 \mathrm{~m}$ wide, a stationary lane having a width of $3.50 \mathrm{~m}$ and two safety lanes having a width of $0.50 \mathrm{~m}$ each. The overall width of the superstructure is $13.60 \mathrm{~m}$ and it consists of the width of carriageway plus two $80 \mathrm{~cm}$ space for the arrangement of deformable safety parapets of heavy type, one on each side.

The viaduct infrastructure is by 2 abutment and 4 piers for each direction of movement. The viaduct superstructure is formed in the cross direction by $4 \mathrm{U}$ prefabricated beams placed at a distance of $3.32 \mathrm{~m}$ between axles, over the beams there is cast a concrete plate of $25 \mathrm{~cm}$ thickness. The beams are made of prestressed concrete class $\mathrm{C} 35 / 45$, and the top plate concrete class $\mathrm{C} 25 / 30$. The viaduct has five equal openings of $36.10 \mathrm{~m}$. The superstructure leaning on infrastructure elements collected and cells was done through the neoprene support devices, Freyssinet type, with a height of $81 \mathrm{~mm}$ [2], and with the critical damping up to a maximum of $10-15 \%$.

\section{Analysis of the dynamic behavior of a bridge section loaded by impulsive actions}

This paper aims at developing a physical and mathematical theory model able to accurately indicate the optimal time for replacing the insulation dynamic systems through periodic monitoring of functional parameters. In this way a section of bridge deck can be considered a solid rigid with viscoelastic tri-orthogonal type links [3].

The matrix expression [1] of the equation that characterizes oscillatory movement of the system can be written as follows:

$$
\underline{I} \underline{\underline{q}}+\underline{C} \underline{\underline{q}}+\underline{K} \underline{\underline{q}}=\underline{f}
$$

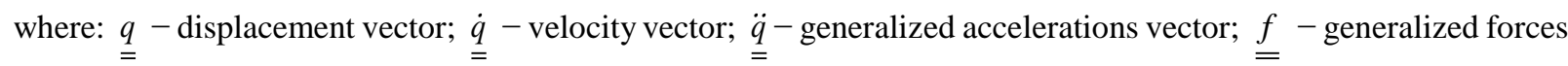
vector; $\underline{I}$ - inertia matrix; $\underline{C}$-damping matrix; $\underline{K}$ - stiffness matrix;

The main elastic axes of elastic bearing are parallel to the reference axes. In this case the system movements, corresponding to the six degrees of freedom, decouple as follows:

- coupled translational motion along the OX axis and axis rotation around the OY axis;

- coupled translational motion along the OY axis and rotation around the OX axis;

- translational motion along the OZ axis independent of the other ways;

- rotation around the $\mathrm{Z}$ axis independent of other modes.

In this case, the system of differential equations can be structured as follows:

a. Coupled mode $\left(\mathrm{X}, \varphi_{\mathrm{y}}\right)$

$$
\left\{\begin{array}{l}
m \ddot{X}+\dot{X} \sum_{1}^{n} c_{i x}+\dot{\phi}_{y} \sum_{1}^{n} z_{i} c_{i x}+X \sum_{1}^{n} k_{i x}+\phi_{y} \sum_{1}^{n} z_{i} k_{i x}=0 \\
J_{y} \ddot{\phi}_{y}+\dot{X} \sum_{1}^{n} z_{i} c_{i x}+\dot{\phi}_{y} \sum_{1}^{n}\left(c_{i z} x_{i}^{2}+c_{i x} z_{i}^{2}\right)+X \sum_{1}^{n} z_{i} k_{i x}+\phi_{y} \sum_{1}^{n}\left(k_{z} x_{i}^{2}+k_{x} z_{i}^{2}\right)=e_{x} F_{z}
\end{array}\right.
$$

where $n$ is the number of bearings, $n=16$. 
b. Coupled mode $\left(Y, \phi_{x}\right)$

$$
\left\{\begin{array}{l}
m \ddot{Y}+\dot{Y} \sum_{1}^{16} c_{i y}-\dot{\phi}_{x} \sum_{1}^{16} c_{i y} z_{i}+Y \sum_{1}^{16} k_{i y}-\phi_{x} \sum_{1}^{16} k_{i y} z_{i}=0 \\
J_{x} \ddot{\phi}_{x}-\dot{Y} \sum_{1}^{16} z_{i} c_{i y}+\dot{\phi}_{x} \sum_{1}^{16}\left(c_{i y} z_{i}^{2}+c_{i z} y_{i}^{2}\right)-Y \sum_{1}^{16} z_{i} k_{i y}+\phi_{x} \sum_{1}^{16}\left(k_{i y} z_{i}^{2}+k_{i z} y_{i}^{2}\right)=-e_{y} F
\end{array}\right.
$$

\section{c. Translation axis $\mathrm{OZ}$}

The fundamental frequency of the bridge deck is $16.3 \mathrm{~Hz}$ on the vertical direction (1).

$$
m \ddot{Z}+\dot{Z} \sum_{1}^{n} c_{i z}+Z \sum_{1}^{n} k_{i z}=-F_{z}
$$

d. Rotation around axis $\mathrm{OZ}$

$$
J_{z} \ddot{\phi}_{z}+\dot{\phi}_{z} \sum_{1}^{n}\left(c_{i x} y_{i}^{2}+2 c_{i y} x_{i}^{2}\right)+\phi_{z} \sum_{1}^{n}\left(k_{i x} y_{i}^{2}+2 k_{i y} x_{i}^{2}\right)=0
$$

where: $m$-deck bridge mass;

$c_{i z}$ - damped coefficient of bearing $i$ on vertical direction;

$k_{i z}$ - coefficient of elasticity of bearing $i$ on vertical direction;

$\mathrm{X}, \mathrm{Y}, \mathrm{Z}$ - deck bridge displacements on $\mathrm{OX}, \mathrm{OY}$ and $\mathrm{OZ}$ directions;

$\phi_{x}, \phi_{y}$ - mass $\mathrm{m}$ rotations around axes OX and OY;

$x_{i}, y_{i}$ and $z_{i}$ - represent the bearings coordinates;

$J_{x}, J_{y}, J_{x}-$ are the main moments of inertia;

$e_{x}$ and $e_{y}$ - the point coordinates of excitation force application from the mass center o of the deck bridge; $F_{z}$ - excitation force.

From the four decoupled motion modes only the mode that corresponds to movement in the vertical direction $\mathrm{OZ}$ will be dealt with in this work. Each section of the bridge is leaning through 16 dynamic isolation systems made of laminated rubber as show in Figs 1, 2 and 3.

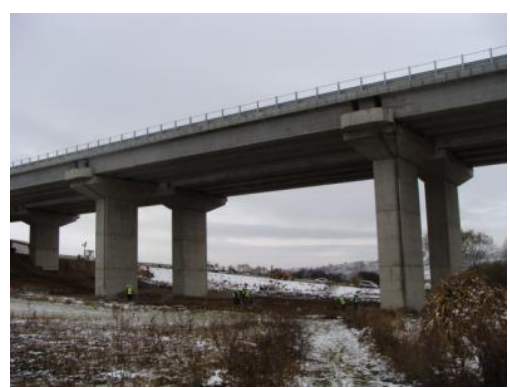

Fig. 1. The bridge deck.

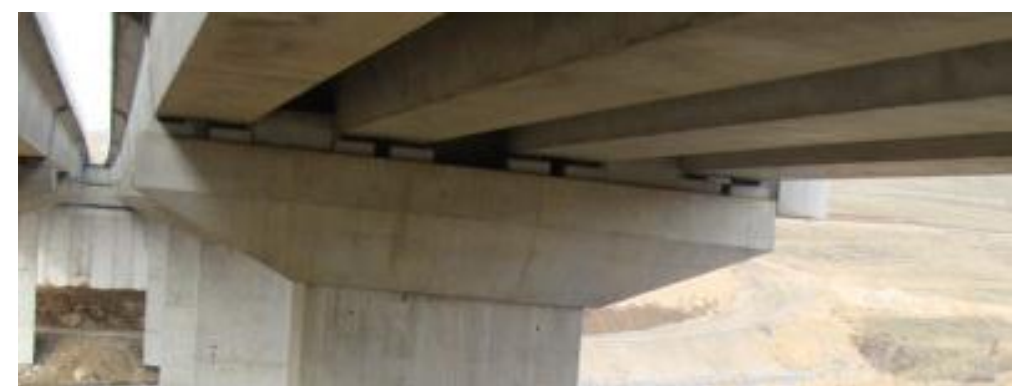

Fig. 2. The bearing systems.

The bridge deck section was impulsively loaded through passing a four-axle truck weighing 41 tons over an obstacle with a height of $40 \mathrm{~mm}(\mathrm{~h}=40 \mathrm{~mm})$, at a speed of $20 \mathrm{~km} / \mathrm{h}$, Fig. 3. The truck passing over the obstacle generates an impulsive loading force which has been considered rectangular shape, as the Fig. 5 shows.

According to Fig. 4, the geometrical and the mass parameters of the vehicle are: $G_{1}=7440 \mathrm{~kg} ; \mathrm{G}_{2}=7339 \mathrm{~kg} ; \mathrm{G}_{3}$ $=13149 \mathrm{~kg} ; \mathrm{G}_{4}=13149 \mathrm{~kg} ; \mathrm{d}_{1}=1.5 \mathrm{~m} ; \mathrm{d}_{2}=2.5 \mathrm{~m} ; \mathrm{d}_{3}=2.0 \mathrm{~m}$. 


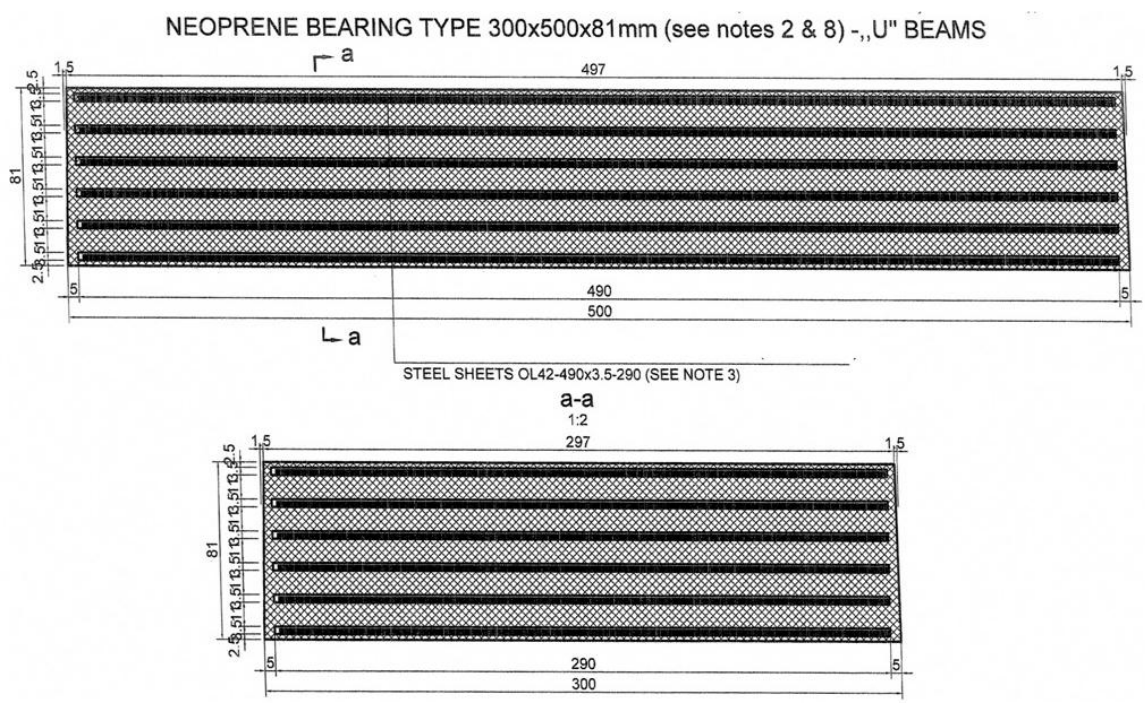

Fig. 3. Neoprene bearing details.

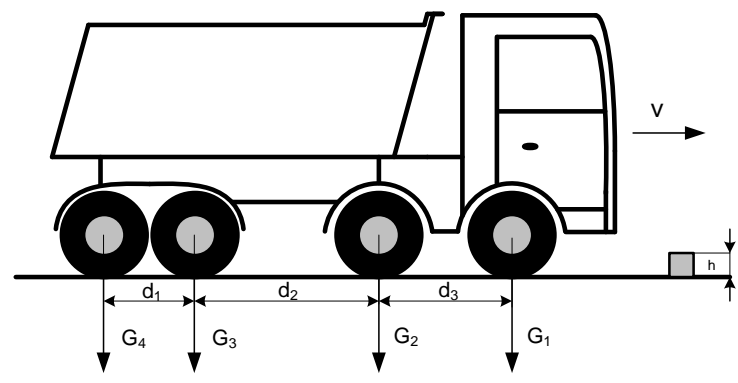

Fig. 4. The simplified model of truck.

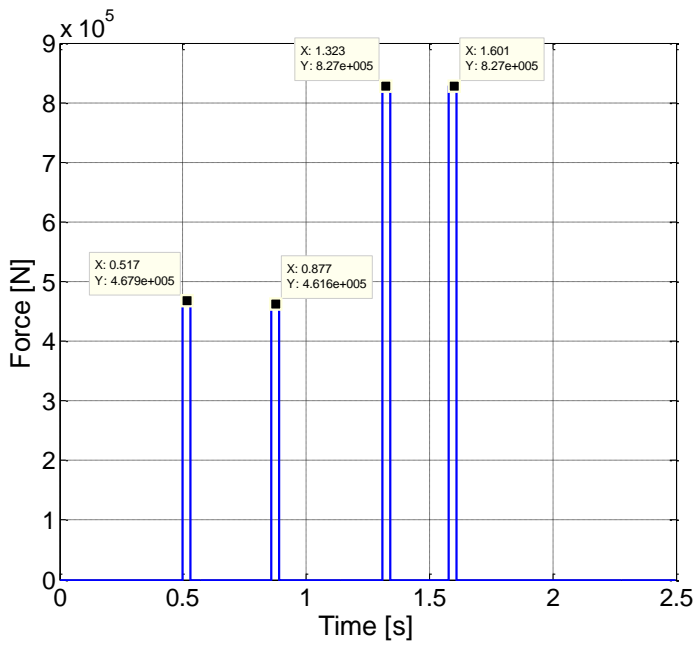

Fig. 5. Four rectangular pulse train.

The rubber bearings wear occurred after the impulsive repeated loadings and atmospheric factors, corresponds to their nonlinear behavior. Thus, two mathematical models corresponding to the linear respectively nonlinear rigidities on $\mathrm{OZ}$ direction will be comparatively analyzed to identify functional parameters that change in the two cases under discussion. The identification of these parameters leads to the establishment of a methodology able to evaluate the degree of structural integrity and optimal function of bearing systems.

In order to determine the degree of degradation/ shading of the viscoelastic links of the anti-earthquake devices based on elastomer, the present paper proposes the following activities:

- at placing the viaduct into function, when the anti-earthquake systems are new and consequently it can be considered that they are characterized by a linear behavior when being compressed, experimental determinations are being made on which a series of parameters of control specific to vibrations are evaluated;

- after a period of time of using the viaduct in normal conditions, the experimental determinations are repeated in the same conditions as those at its placing in function, through which we seek to achieve the quantitative and qualitative characterization of the parameters of control; 
On the basis of those two sets of parameters of control obtained a comparative analysis is achieved in order to highlight the differences that occur between them. The potential differences identified highlight the degradation of the viscoelastic links of the anti-earthquake bearings.

The main management activities [5] for monitoring the technical systems vibration are presented in Fig. 6 .

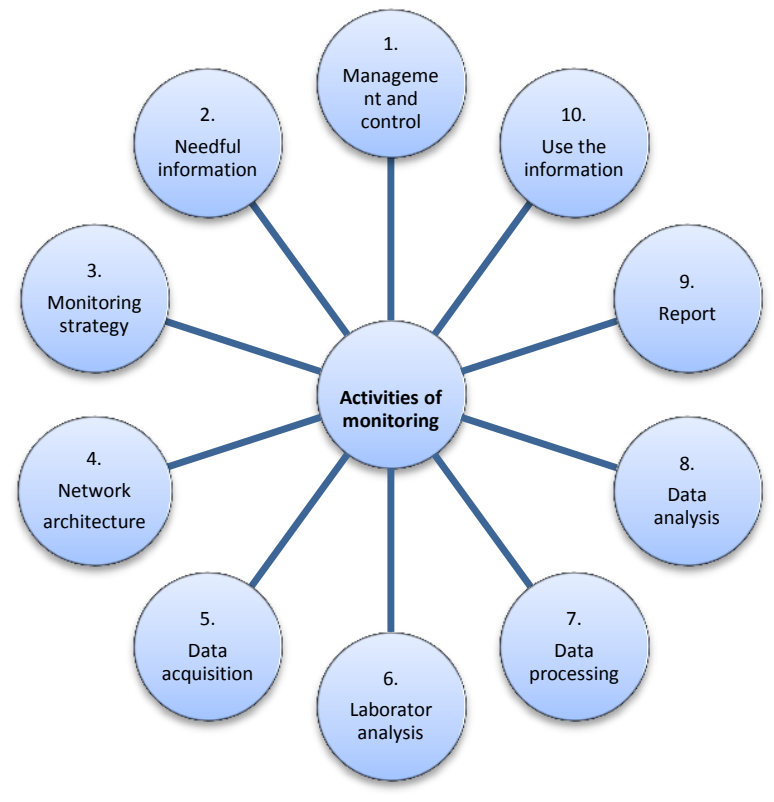

Fig. 6. Activities of monitoring.

\section{Identification and interpretation of kinematics and energetic parameters}

The dynamic response of bridge deck was analyzed after impulsive loading produced from a passing truck weighing 41 tons over an obstacle $40 \mathrm{~mm}$ high, assuming the two kinds of elastic forces of dynamic isolation systems as linear and nonlinear type [3].

\subsection{The case of linear elastic forces}

In this case the equation of motion is as follows [1]:

$$
m \ddot{Z}+\dot{Z} \sum_{1}^{n} c_{i z}+Z \sum_{1}^{n} k_{i z}=-F_{z}
$$

where: $m$ - mass of the deck;

$c_{i z}$ - damped coefficient of bearing $i$ on vertical direction;

$k_{i z}$ - coefficient of elasticity of bearing $i$ on vertical direction;

$Z$ - movement on vertical direction;

$F_{z}$ - vertical direction force application;

$i$ - current number of bearing system.

\subsection{The case of nonlinear elastic forces}

In this case the equation of motion is as follows [6]: 


$$
m \ddot{Z}+\dot{Z} \sum_{1}^{16} c_{i z}+Z \sum_{1}^{16} k_{i z}\left(1+\beta Z^{2}\right)=-F_{z}
$$

The graphic representations of stiffness coefficients for the two considered cases linear and nonlinear are presented in Figs 7 and 8.

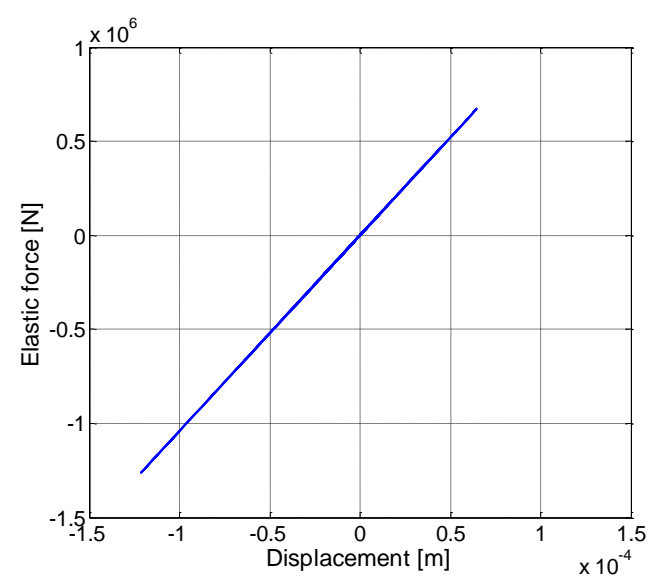

Fig. 7. The representations of stiffness coefficients: Linear case.

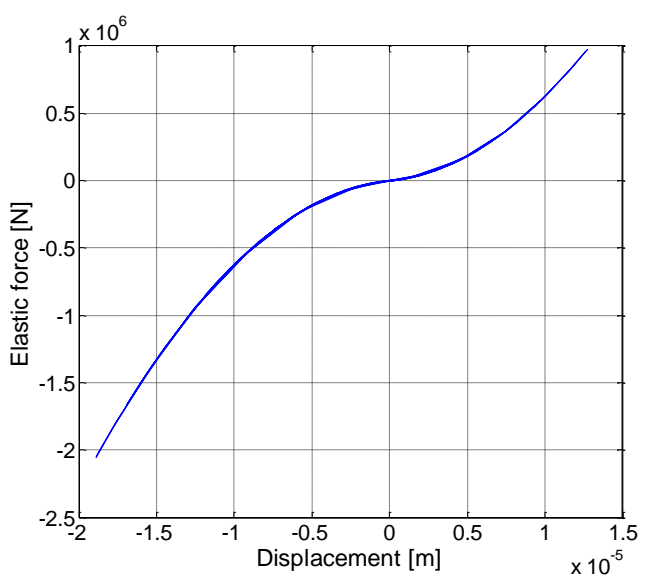

Fig. 8. The representations of stiffness coefficients: Nonlinear case.

Based on the differential equation of motion (7) the specific vibration parameters of the deck with viscoelastic bearing were analyzed and plotted as follows:

- time response of the cinematic parameters of vibration;

- frequency response of the cinematic parameters of vibration;

- energy dissipated by viscous friction;

- motion trajectory;

- power spectral density.

MATLAB program [7] was used for solving mathematical model by assuming the following numerical values of the coefficients of equation of motion $k_{i z}=650 \cdot 10^{6} \mathrm{~N} / \mathrm{m} ; c_{i z}=2.5 \cdot 10^{6} \mathrm{Ns} / \mathrm{m} ; m=992 \cdot 10^{3} \mathrm{~kg} ; \beta=5 \cdot 10^{10} \mathrm{~m}^{-2}$.

Figures 9-20 show the graphical representations of cinematic and energy parameters of the vibration of the bridge deck.

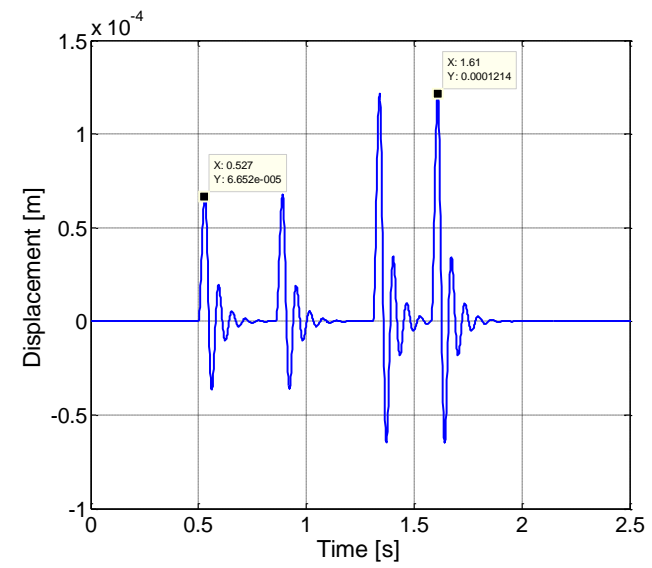

Fig. 9. Displacement on mass m: Linear case.

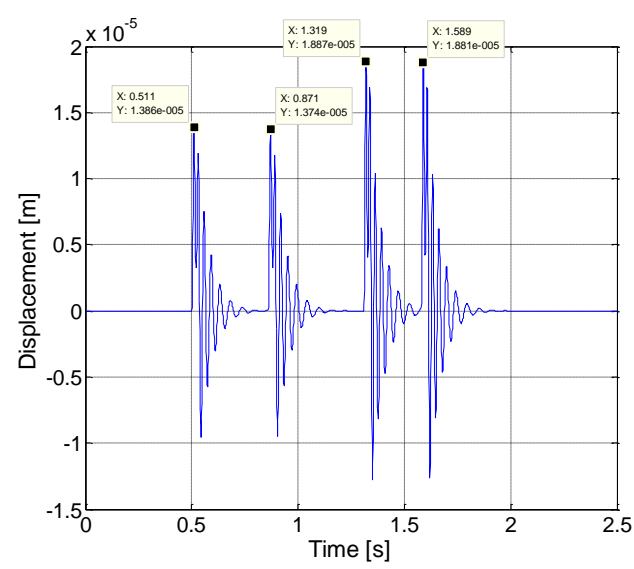

Fig. 10. Displacement on mass m: Nonlinear case. 
From the time domain representation of the displacement of mass $m$ it was observed that in nonlinear elastic forces case, there are both quantitative and qualitative changes, Figs 9 and 10. The displacement amplitude, in nonlinear case decreases to a value of $1.88 \cdot 10^{-5} \mathrm{~m}$. For the nonlinear case, the spectral representation of the displacement of mass $\mathrm{m}$, reveals an increase of the dominant frequency values at $47 \mathrm{~Hz}$, compared to linear case in which the dominant frequency was up to $22.33 \mathrm{~Hz}$. The appearance of supra-harmonics was highlighted as shown in Figs 11 and 12.

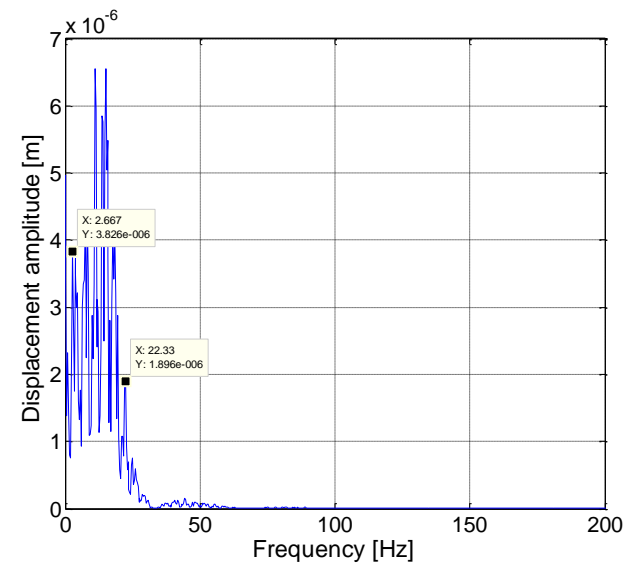

Fig. 11. Spectral representation - linear case.

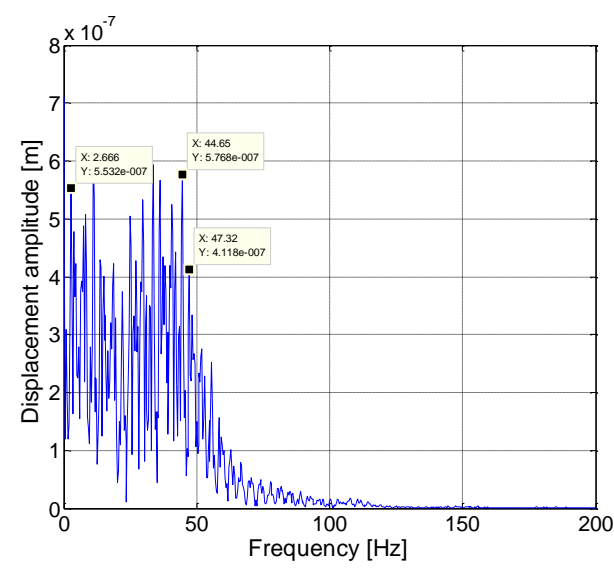

Fig. 12. Spectral representation - nonlinear case.

For the displacement signal, the logarithmic decrement $\Delta$ and the fraction from the critical amortization $\zeta$ have been determined, corresponding to significant frequencies, Tables 1 and 2.

The logarithmic decrement and critical damping fraction have different values in the two considered cases, because for nonlinear antiseismic system, the damping capacity is different compared to the linear case.

In Tables 1 and 2 used the following were notations:

$f_{j}-$ the frequency of the $j$ order vibration;

$\Delta$ - the logarithmic decrement;

$\zeta$ - the fraction of critical amortization;

$\tilde{\mathrm{A}}$ - the effective value (R.M.S) of the amplitude of displacement (from the spectrum of movement).

Table 1

The vibration parameters - linear case

Linear vibration case

\begin{tabular}{cccc}
\hline \multicolumn{4}{c}{ Linear vibration case } \\
$\tilde{A}$ & \multicolumn{3}{c}{ The parameters of fundamental vibration } \\
\cline { 2 - 4 }$(\mu \mathrm{m})$ & $\mathrm{f}_{\mathrm{j}}$ & $\Delta$ & $\zeta$ \\
\hline 3.82 & 2.66 & 0.026 & 0.41 \\
6.54 & 11 & 0.11 & 1.76 \\
6.55 & 15 & 0.48 & 7.72 \\
\hline
\end{tabular}

Table 2

The vibration parameters - nonlinear case

\begin{tabular}{cccc}
\hline \multicolumn{4}{c}{$\begin{array}{c}\text { Nonlinear vibration case } \\
\text { The parameters of fundamental vibration }\end{array}$} \\
\cline { 2 - 4 }$\tilde{\mathrm{A}}$ & $\mathrm{f}_{\mathrm{j}}$ & $\Delta$ & $\zeta$ \\
$(\mu \mathrm{m})$ & $(\mathrm{Hz})$ & & $(\%)$ \\
\hline 0.55 & 2.66 & 0.139 & 2.22 \\
0.60 & 11 & 0.33 & 5.35 \\
0.59 & 33.6 & 0.039 & 0.64 \\
\hline
\end{tabular}

From the analysis of acceleration signals for the two cases, Figs 13 and 14, a noticeable increase of the maximum value was observed, from $1.3 \mathrm{~m} / \mathrm{s}^{2}$ in linear case to $2 \mathrm{~m} / \mathrm{s}^{2}$ in case nonlinear, as well as a shape distortion. The spectral representation of acceleration reveals, as in the movement case, a broadening of the domain of dominant frequency band spectral components, Figs 15 and 16. 


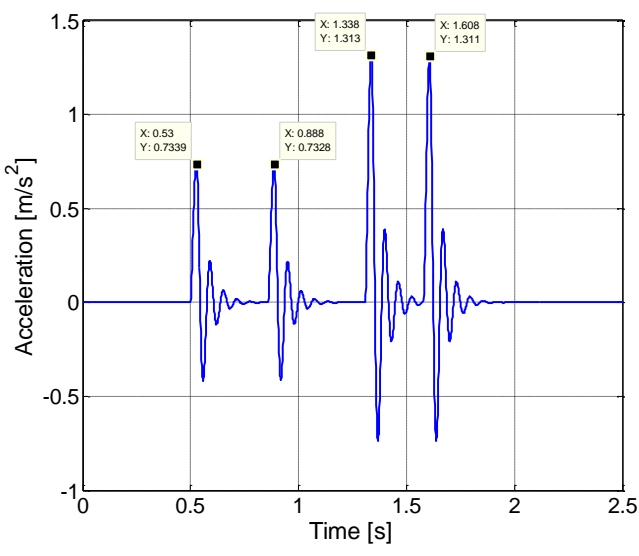

Fig. 13. Acceleration of mass $m$ - linear case.

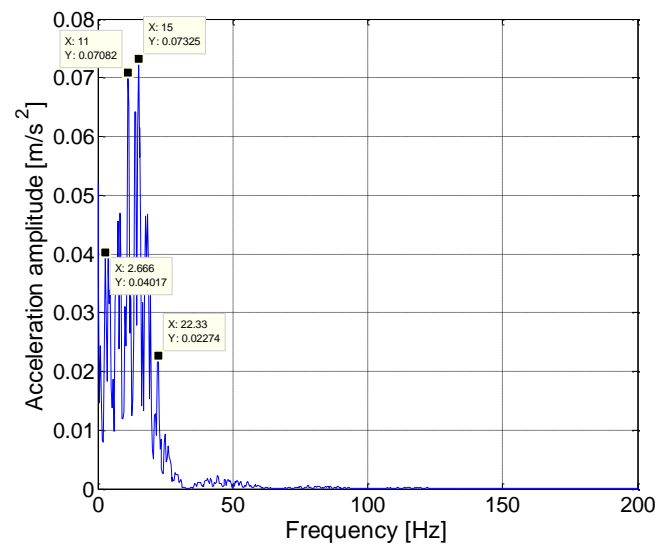

Fig. 15. Spectral representation - linear case.

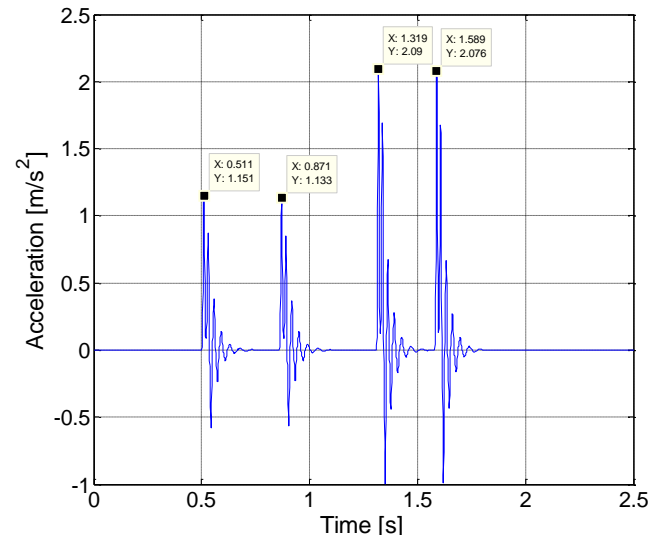

Fig. 14. Acceleration of mass $m-$ nonlinear case.

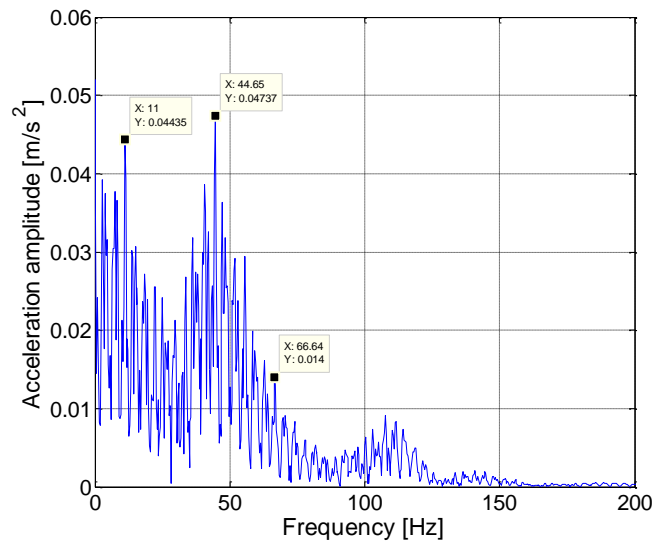

Fig. 16. Spectral representation - nonlinear case.

The hysteretic loops have as symmetry axes the graphical representations of the elasticity coefficients, the energy dissipated in the same time interval being different, Figs 17 and 18. In the nonlinear elastic force case a decrease of the value dissipated energy to $20 \mathrm{~J}$ was observed (12 times less than in the linear case), which means that a greater quantity of energy remains in the system.

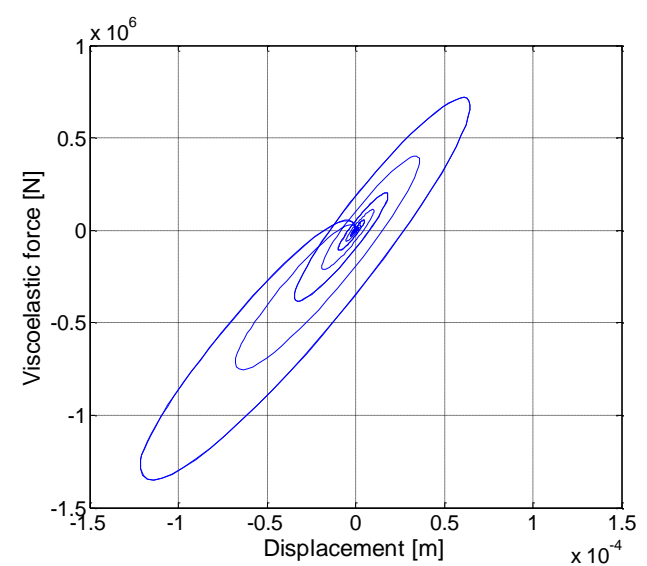

Fig. 17. Hysteretic loop: Llinear case, $\mathrm{W}=256 \mathrm{~J}$.

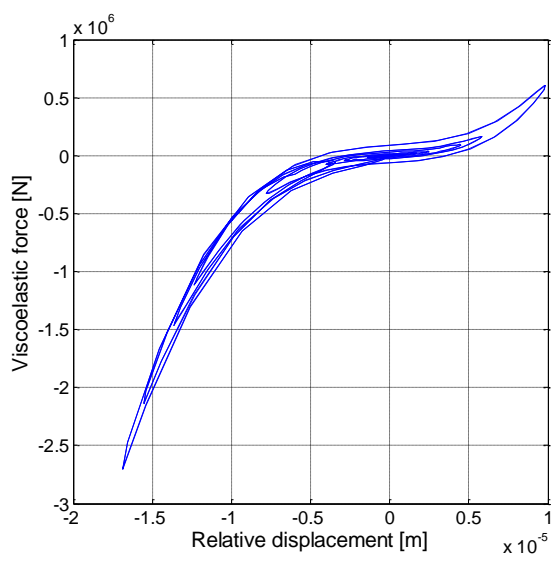

Fig. 18. Hysteretic loops: Nnonlinear case, $\mathrm{W}=20 \mathrm{~J}$. 


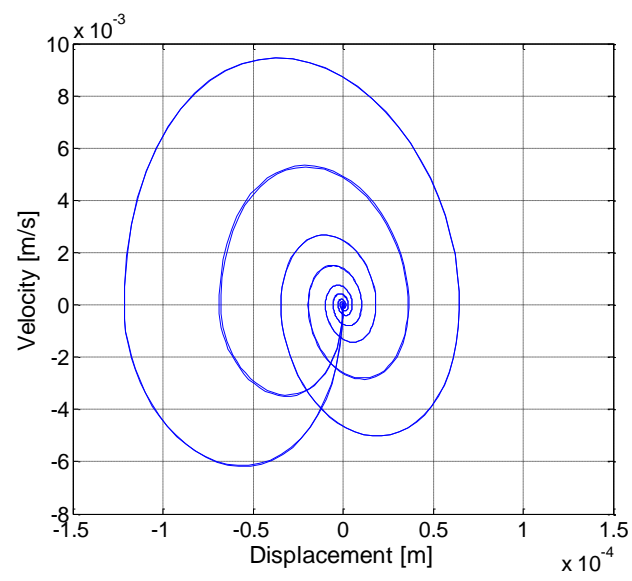

Fig. 19. Phase plane representation: Llinear case.

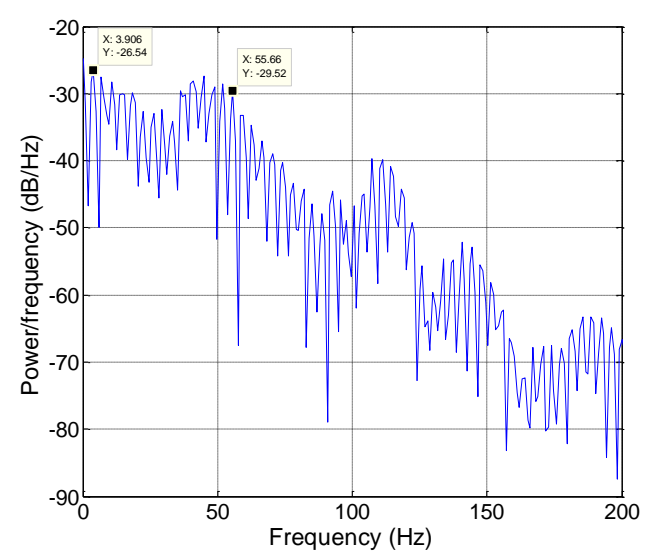

Fig. 21. Periodogram: Llinear case.

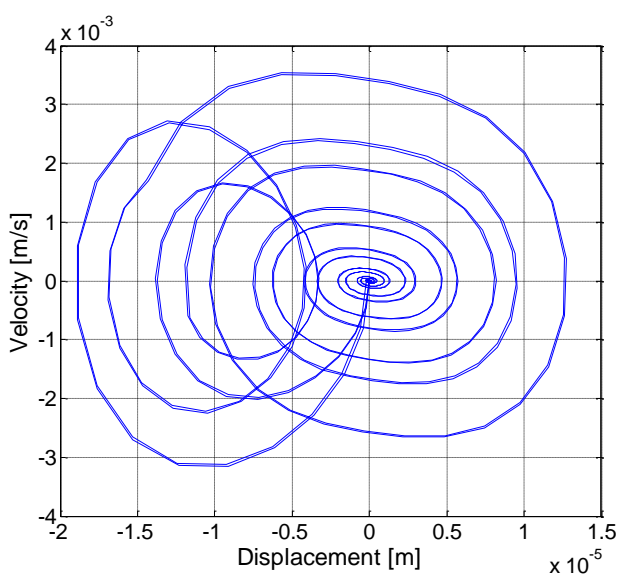

Fig. 20. Phase plane representation: Nnonlinear case.

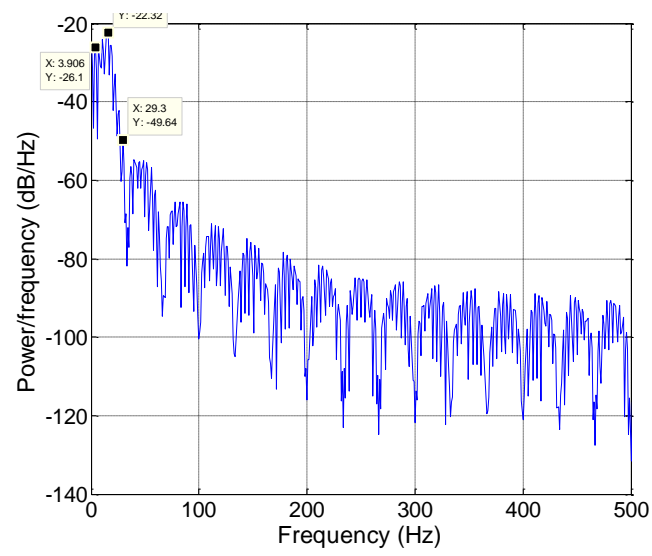

Fig. 22. Periodogram: Nnonlinear case.

The phase plane representation, Figs 19, 20, shows an additional motion around a strange attractor point for the case of nonlinear elastic forces. The power spectral density graphics for the two analyzed cases, Figs 21, 22, reveal that in the nonlinear case a significant value of average power of the signal is carried by spectral components in the range of frequency $(0 \div 55.66) \mathrm{Hz}$, whereas in the linear case this interval is $(0 \div 29.3) \mathrm{Hz}$.

An useful representation to identify spectral participation of each excitation pulse is the spectrogram of signal displacement, presented in several forms in Figs 23-26.

This kind of representation proves its usefulness especially in cases where the mechanical system load is made by multiple excitations. It is noted that for high frequency vibrations the excitations three and four from the train of pulses are responsible.

\section{Experimental results}

It is obvious that a theoretical model shall be validated by experimental tests, for confirming the possibility of using it with as little error. In this paper, from an experimental point of view, the parameters of control of normal functioning for anti-earthquake systems will be quantified on placing in function of the viaduct on the highway Transilvania, Romania. Thus, qualitative and quantitative evaluations are obtained and they will serve as reference for the same parameters determined at further moments. 


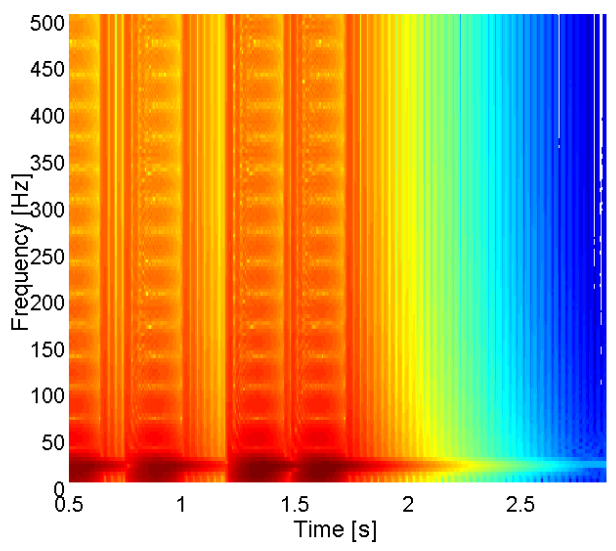

Fig. 23. 2D spectrogram of acceleration signal: Linear case.

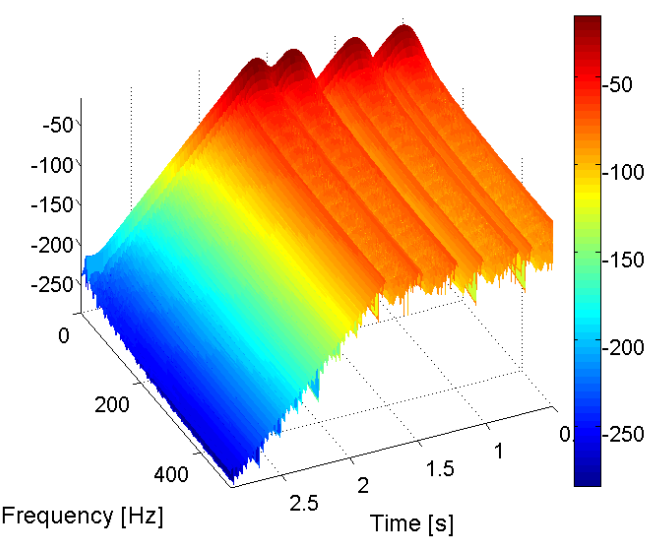

Fig. 25. 3D spectrogram of acceleration signal: Linear case.

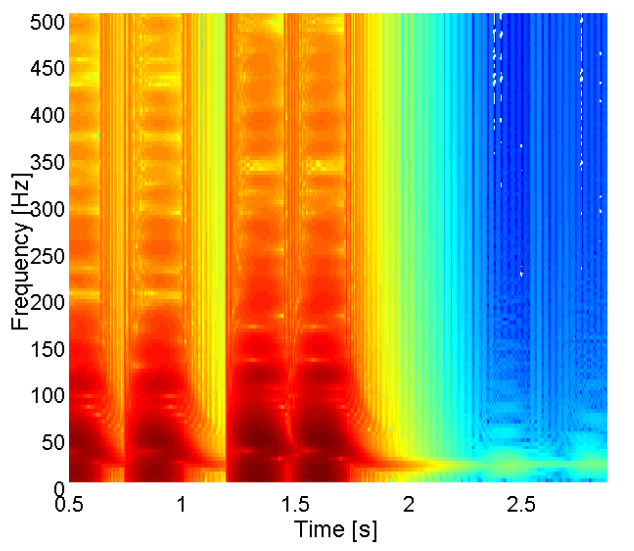

Fig. 24. 2D spectrogram of acceleration signal: Nonlinear case.

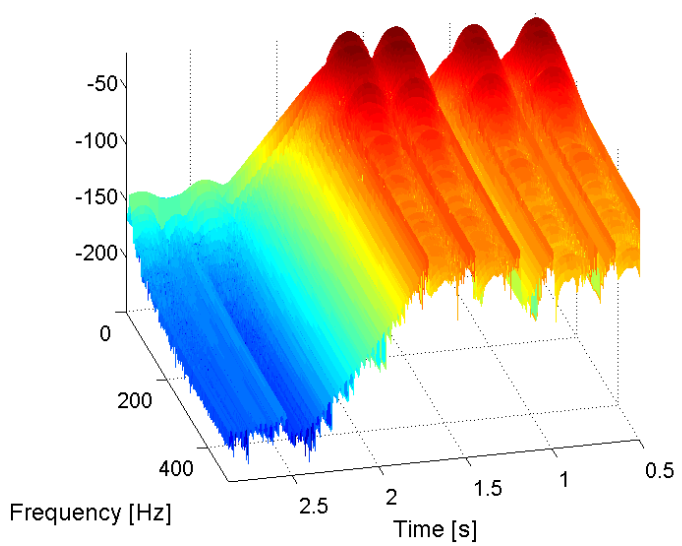

Fig. 26. 3D spectrogram of acceleration signal: Nonlinear case.

Thus, experimental measurements were made under the same conditions previous presented, using a triaxial accelerometer Bruel\&Kjaer type 0034506 B series 10145 positioned in the transverse plane of symmetry [2]. Triaxial accelerometer Bruel\&Kjaer has the following dynamic characteristics:

- Sensitivity (at $159.2 \mathrm{~Hz}): 50 \mathrm{mV} / \mathrm{ms}^{-}-2 \pm 10 \%,(500 \mathrm{mV} / \mathrm{g}+8,-12 \%)$

- Measuring Range: $\pm 140 \mathrm{~ms}-2(14 \mathrm{~g})$

- Frequency Range: $( \pm 10 \%)$ : X: $0.3 \mathrm{~Hz}$ to $4.0 \mathrm{kHz}$; Y, Z: $0.3 \mathrm{~Hz}$ to $2.0 \mathrm{kHz}$

- Phase Response: $2 \mathrm{~Hz}$ to $2.5 \mathrm{kHz}, \pm 5^{\circ}$

- Mounted Resonance Frequency: X: 14.0 kHz; Y, Z: 7.0 kHz

The accelerometer was positioned to measure data on the following:

a. OX axis - longitudinal direction

b. OY axis - transverse direction

c. $\mathrm{OZ}$ axis - vertical direction.

Furthermore only the parameters of the vibration corresponding to axis $\mathrm{OZ}$ will be analyzed, because on this direction the highest values of acceleration have been identified.

\subsection{Graphical representation of acceleration in the OZ direction}

The acceleration signal acquired has been filtered in order to get away of the background noise using Wavelet 
toolbox denoising [7] from the software MATLAB software packet, Fig. 27. A detailed analysis of the residual signal is particularly useful, by providing information on sources of vibration that accompanies good signal.

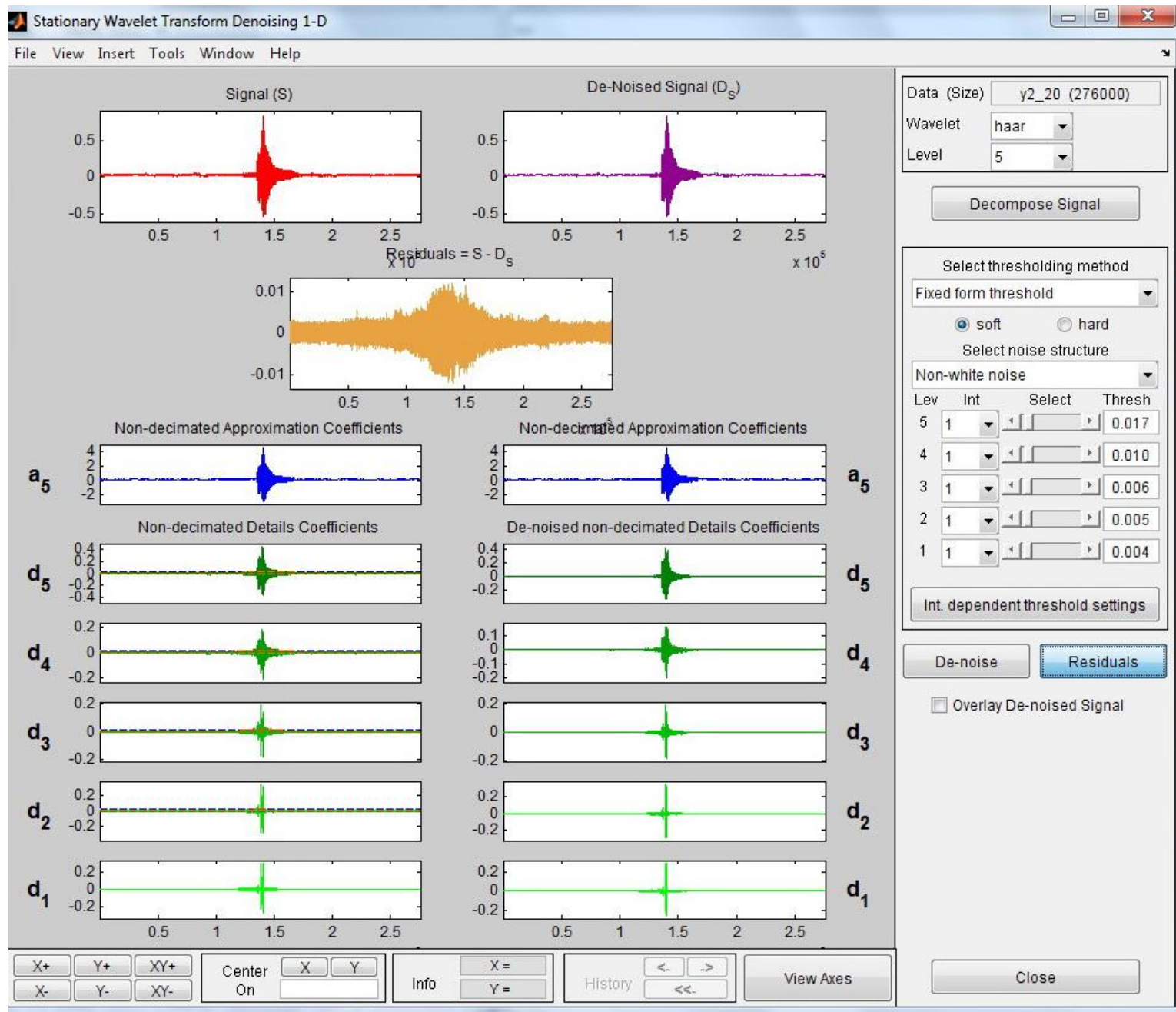

Fig. 27. The filtering of the acceleration signal experimentally acquired.

The analysis of the residual signal (Fig. 28), in the time and frequency domains provides useful information on the sources of parasitic vibrations which constitutes a criterion in the adjustment and choosing the range of measurement and control devices.

The maximum acceleration value of $0.8 \mathrm{~m} / \mathrm{s}^{2}$ is obtained by passing of the vehicle axle with highest load, Fig. 29 . From the spectral representations of acceleration in $\mathrm{OZ}$ direction one can observe an interval of the dominant spectral components similar to the corresponding linear case 4.1-12.5 Hz, Fig. 30.

It should be noted that when performing the experimental measurements the dynamic isolation systems were new and consequently their characteristics can be considered linear.

Power spectral density shows a significant distribution of signal strength on the frequency range $0-35 \mathrm{~Hz}$, aspect that has been identified from spectral representation of the acceleration signal, Fig. 31.

The spectrogram acceleration in the $\mathrm{OZ}$ direction is another parameter able to monitor the state of normality in the functioning of bearing systems, Figs 32 and 33. In this case, 2D and 3D representation are similar with the same representation of the linear case. 


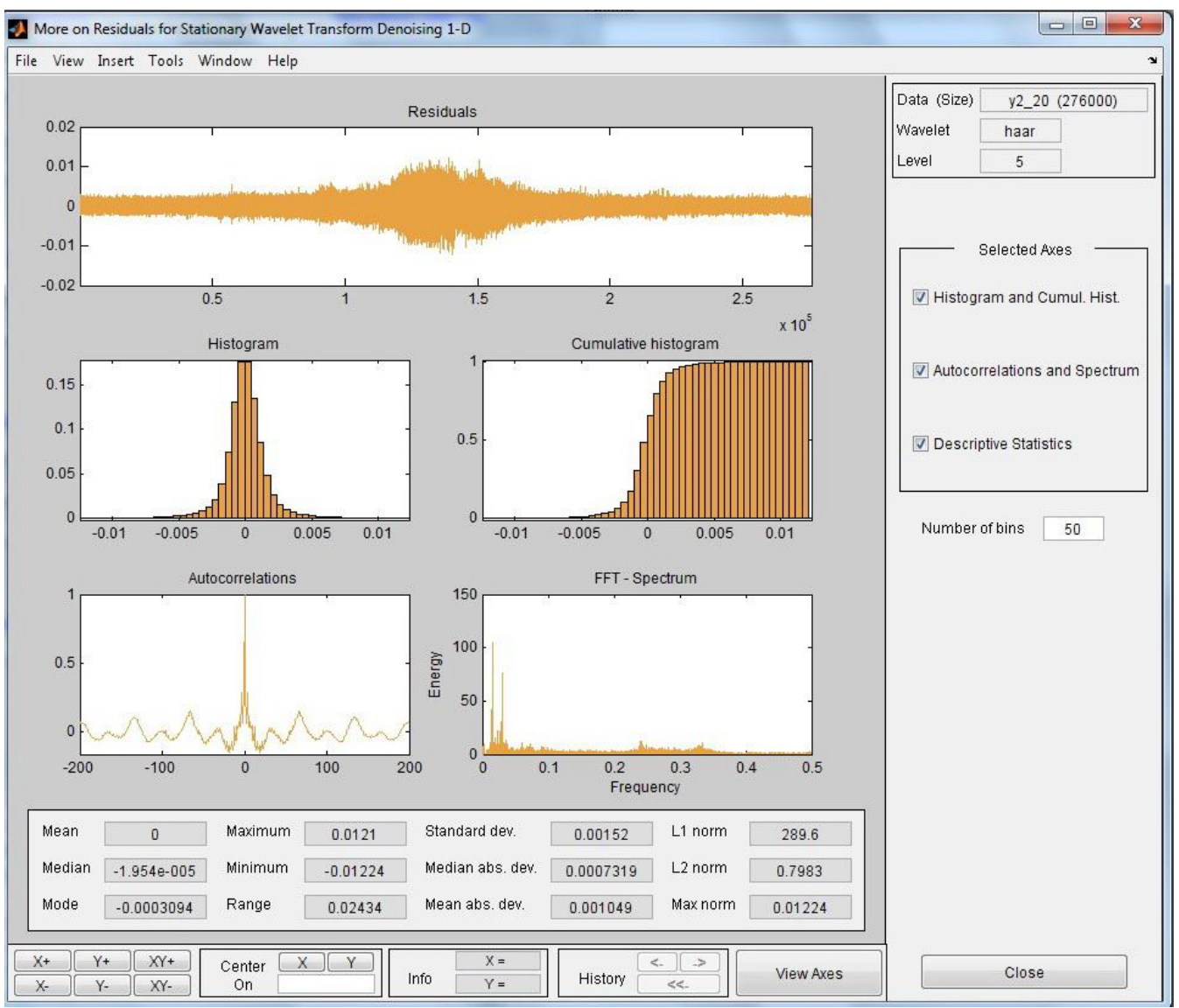

Fig. 28. The residual signal.

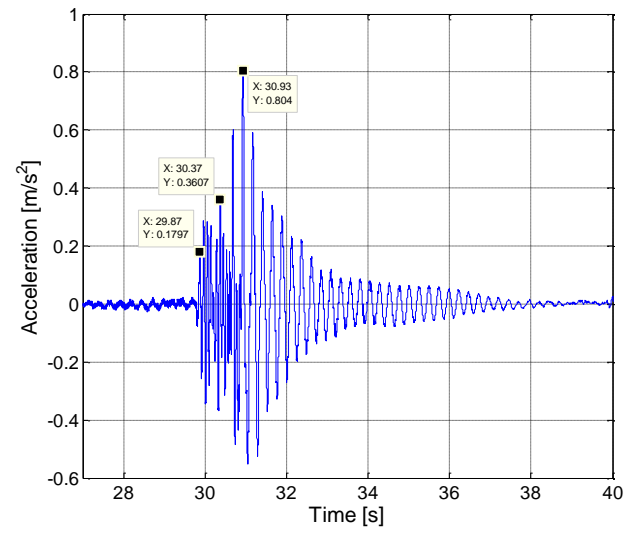

Fig. 29. Acceleration on $\mathrm{OZ}$ direction.

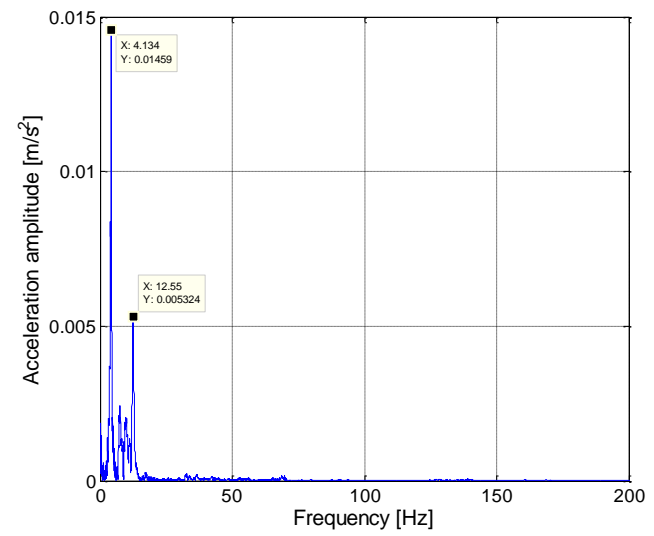

Fig. 30. Spectral representation.

\section{Conclusion}

The inefficiency of dynamics insulation systems occurred after seismic activities or road or rail traffic, may gradually affect the structural integrity of the deck bridge or in extreme cases even destroy it. For this reason, this 


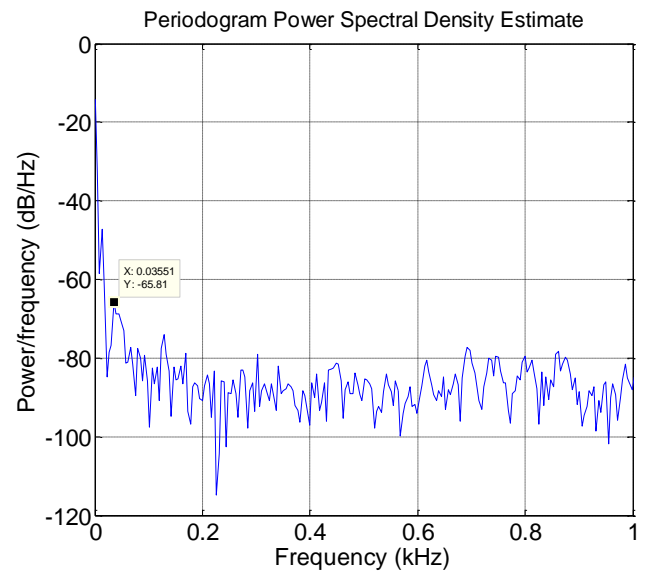

Fig. 31. Acceleration on $\mathrm{OZ}$ direction periodogram.

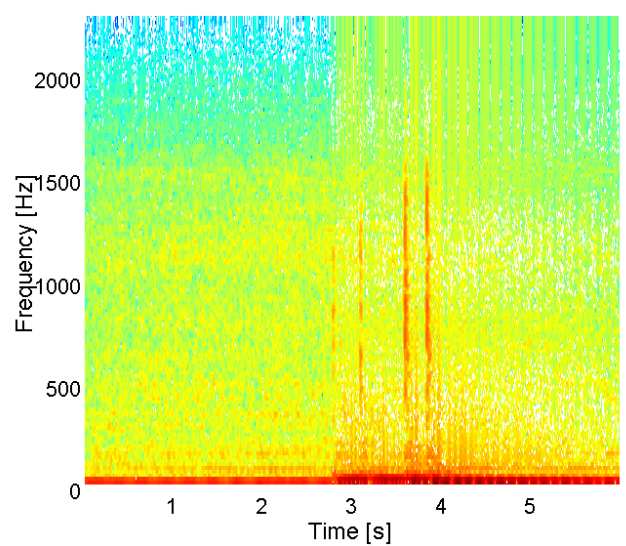

Fig. 32. 2D spectrogram of acceleration signal.

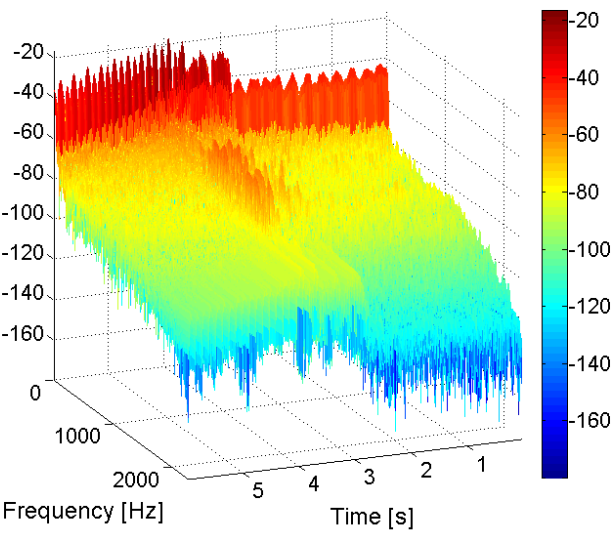

Fig. 33, 3D spectrogram of acceleration signal.

paper proposes an effective methodology for evaluation of the degradation of dynamic isolation systems based on rubber molding, by comparing, in different periods of time, of the parameters able to highlight the changes that occur in the dynamic response of the structure after impulsive loading.

These correlations between the nonlinear behaviour of the elastic component of the bearing systems and dynamic changes that occur in the response of the structure were highlighted only theoretically, but in this way open the opportunity for the experimental study of this theory.

From the theoretical study developed in this paper the following actual aspects are highlighted:

- The representation of the displacement in the time domain highlights a decrease of its amplitude from $1.2 \cdot 10^{-4} \mathrm{~m}$ in a linear case to $1.88 \cdot 10^{-5} \mathrm{~m}$ in the nonlinear case, which demonstrates an increase of the stiffness of the anti- earthquake system on upright direction.

- The spectral representation of the displacement highlights a decrease of the band of dominant frequencies in nonlinear case focused on the value of $22 \mathrm{~Hz}$, whereas in the linear case the central value is of $15 \mathrm{~Hz}$. This modification proves the modification of the rheological parameters of the systems of anti- earthquake bearing.

- The representation of the acceleration in the time domain highlight a increase of its amplitude from $1.3 \mathrm{~m} / \mathrm{s}^{2}$ in linear case to $2 \mathrm{~m} / \mathrm{s}^{2}$ in nonlinear case, which proves an increase of the stiffness of the anti- earthquake system on upright direction. 
- the spectral representation of the acceleration highlights an increase of the band of dominant frequencies in nonlinear case with values focused on 11 and $44.65 \mathrm{~Hz}$, whereas in linear case the central values are $11 \mathrm{~Hz}$ and $15 \mathrm{~Hz}$.

- The representation of the hysteretic loop highlights a decrease of almost 10 times of the energy dissipated by the anti-earthquake systems, which leads to the presence of a remaining energy in the system.

- The power spectral density highlights an increased interval in non-linear case on which the power of the signal is delivered $(0 \div 55.66) \mathrm{Hz}$, whereas for the linear case this domain is of $(0 \div 29.3) \mathrm{Hz}$.

The differences identified between the values and graphic representations of the parameters of control for the two cases in point (linear and non-linear), prove the modification of the rheological characteristics of the bearings systems and consequently the modification of their viscoelastic links.

From an experimental point of view the parameters of control of vibration have been quantified on placing the bridge in function. Experimentally, in order to complete this study in the future, there is the intention of performing some experimental tests in the same initial conditions, in order to determine and comparatively analyse the parameters of control.

\section{Acknowledgments}

This work was supported by UEFISCDI (CNCSIS-UEFISCSU), project number PN II-RU-PD code 597/2010.

\section{References}

[1] N. Dragan, Analiza experimentală a dinamicii podurilor din beton armat supuse acţiunilor din trafic, SINUC, Bucuresti, 2010.

[2] Final report on testing for Transylvania highway viaduct, ICECON SA, 2009.

[3] A. Leopa and S. Nastac, Dynamic behaviour of foundations in linear and nonlinear elastic characteristics hypothesis, WSEAS Transactions on Applied and Theoretical Mechanics 4(3) (2008), 145-154.

[4] A. Leopa and C. Debeleac, On shock inertial excitation methods for concrete viaduct dynamic testing, The 10th International Conference on Vibration Control, Czech Republic, 2011, pp. 57-62.

[5] A. Leopa, Evaluation of environmental pollution by mechanical vibrations from the technological equipment with anti-vibration systems with degraded viscoelastic links, Journal of Science and Arts 1(12) (2010), 67-72.

[6] A. Leopa, S. Nastac, N. Dragan and C. Debeleac, Considerations on the influence of viscoelastic behavior of nonlinear systems bearing on the dynamic constructions response, International Conference on Structural Engineering Dynamics, Tavira, Portugalia, 2011.

[7] MATLAB, High-Performance Numeric Computation and Visualisation Software. Reference Guide, The MATWORKS Inc., Natick, Massachusetts, 1992.

[8] J. Awrejcewicz and R. Andrzejewski, Nonlinear Dynamics of a Wheeled Vehicle, Springer - Verlag, Berlin 2005.

[9] J. Awrejcewicz and L. Dzyubak, Influence of hysteretic dissipation on chaotic responses, Journal of Sound and Vibration 284 (2005), 513-519.

[10] J. Awrejcewicz, K. Tomczak and C.-H. Lamarque, Controlling system with impacts, International Journal of Bifurcation and Chaos 9(3) (1999), 547-553. 

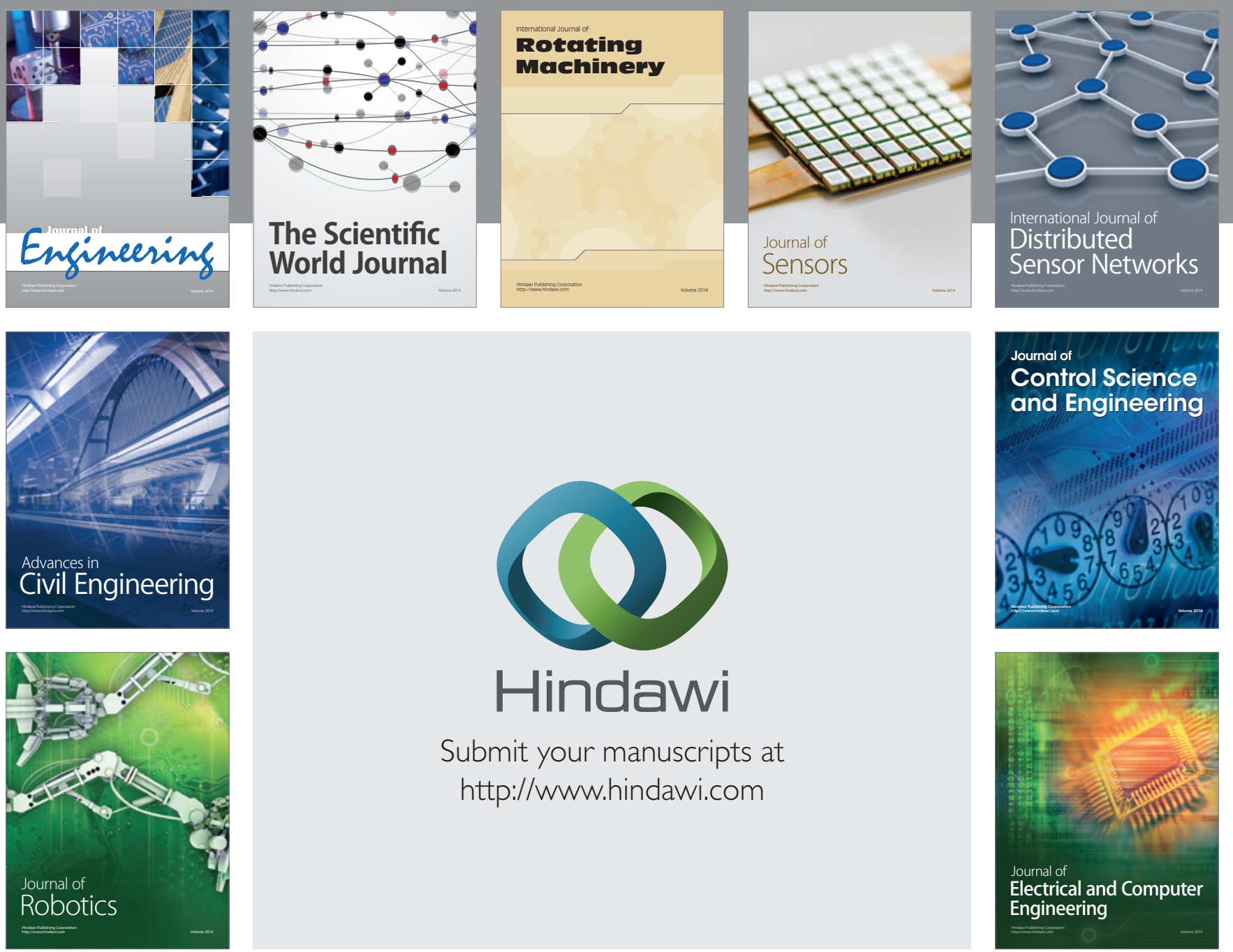

Submit your manuscripts at

http://www.hindawi.com
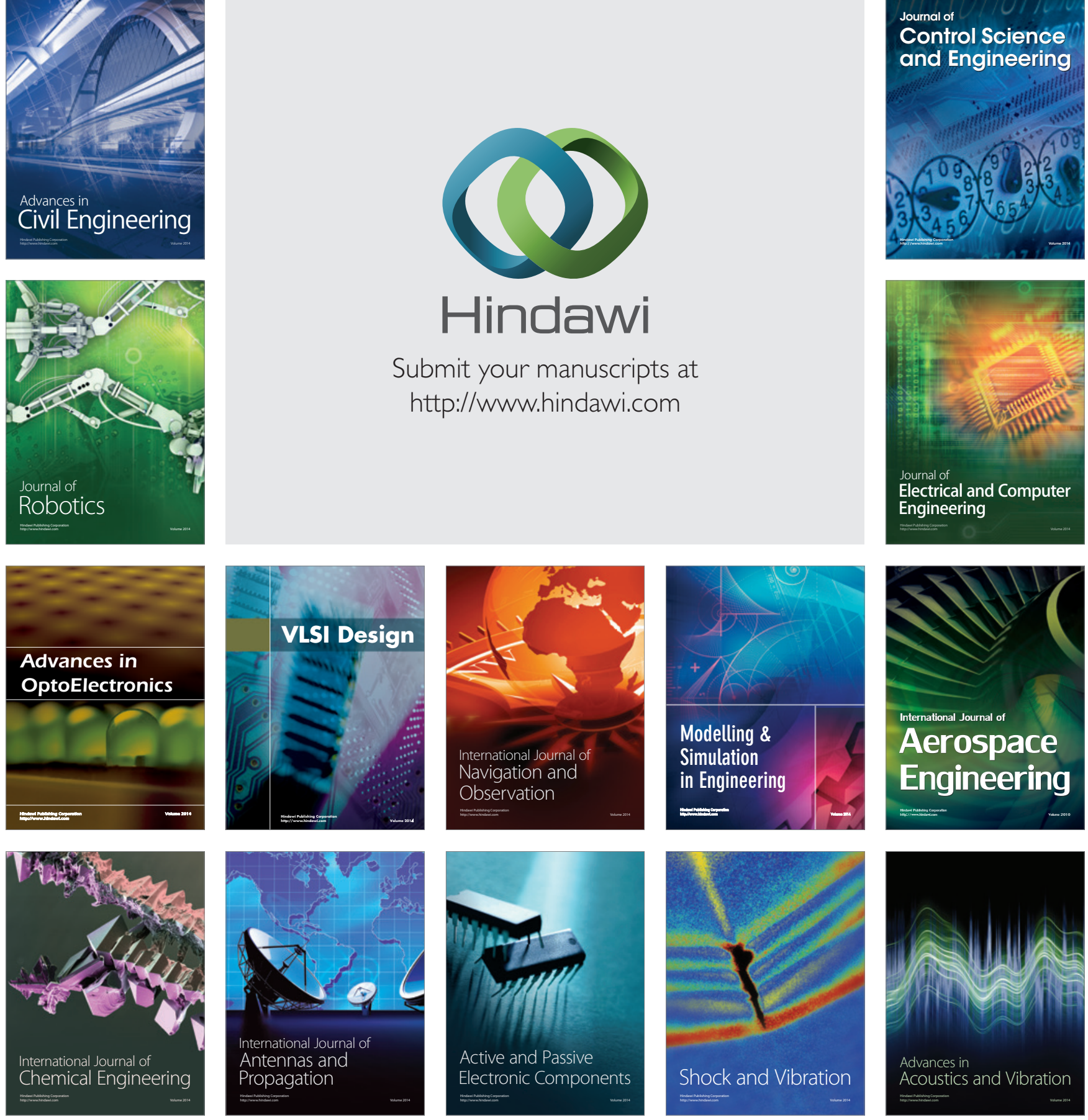